\title{
Calculations on 2-dimensional Waveguides in Photonic Crystals
}

\author{
R. Stoffer, R.M. de Ridder, H.J.W.M. Hoekstra, E.W.C. van Groesen, F.P.H. van Beckum, and A. Driessen \\ University of Twente, MESA Research Institute, PO Box 217, 7500 AE Enschede, the Netherlands, \\ E-mail: R.M.deRidder@el.utwente.nl
}

\begin{abstract}
Using 2 different numerical methods (FDTD and a novel pandirectional planar Helmholtz solver), we analyzed the waveguide behaviour of a channel in a 2-dimensional photonic crystal including its dispersion and coupling to incoming waves.
\end{abstract}

Photonic crystals [1] can be considered as generalized gratings in 1,2 or 3 dimensions. These periodic structures, having a period in the order of a wavelength have the important property that electromagnetic waves in a certain frequency range cannot propagate in any direction in the structure, hence they are often referred to as photonic bandgap materials. In order to obtain wide and overlapping bandgaps for TE and TM polarizations, a relatively large $(>\sim 2)$ refractive-index contrast is needed. If a channel is formed in such a crystal (e.g. by removing a row of elements), it can act as a highly efficient and compact waveguide for frequencies in the bandgap. Since no energy of such waves can radiate away through the crystal, waveguides in the crystals can have low-loss bends with extremely small bending radius [2]. We believe that such waveguides are a key to ultra high density opto-electronic integration which will be needed e.g. for the fabrication of large optical switching matrices or chip-level optical interconnects.

In order to design waveguides and other devices in photonic crystals, more is needed than the well-known bandstructure calculations for perfectly regular crystals. For investigating propagation and localization of electromagnetic fields, a modeling tool is needed which can account for pandirectional propagation. This precludes using the well-known and efficient beam propagation methods (BPM's) [3]. Given the computing power of presentday personal computers, we restricted our computations to 2 dimensions, using the well-known finite-difference time-domain (FDTD) method [4] and a novel method for solving the Helmholtz equations in a plane [5], both implemented using boundary conditions based on the perfectly-matched layer [6] technique. We will illustrate the flexibility of these methods with a few examples. It should be noted that a full 3D-analysis will be required for accurately modeling radiation phenomena perpendicular to the plane of the waveguides, in particular if the limited thickness of practical photonic crystals is to be taken into account.

Figure 1 shows a method for determining the bandgap of a $2 \mathrm{D}$ photonic crystal (here a rectangular array of parallel high-index, square crosssection rods). A dipole, radiating in all directions in the plane, is driven with a very short optical pulse covering a broad wavelength spectrum of 0.7-2.5 $\mu \mathrm{m}$. The transmission of the wave to each point at the right side of the crystal is calculated with FDTD (for symmetry reasons an angular range of only $45^{\circ}$ needs to be considered). Then, the Fourier transform of the transmitted pulse is taken, giving the transmission spectrum for each point (direction). The right part of fig. 1 is the superposition of all these spectra, showing a clear
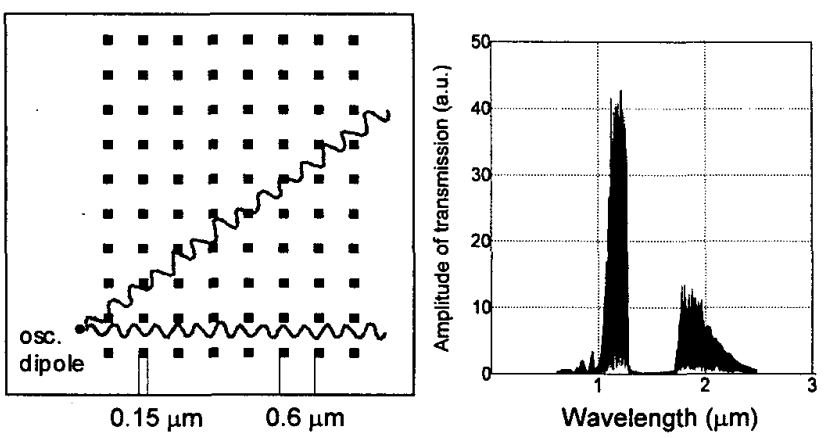

Fig. 1 Bandgap calculation. Left: crystal and dipole radiator. Right: superposition of transmission spectra, showing the bandgap. bandgap of approximately 1.3-1.7 $\mu \mathrm{m}$.

The dispersion of a waveguide can be calculated (fig. 2) by terminating it with a perfect reflector (such as a photonic crystal), which gives rise to a standing-wave pattern in the guide. From the distance between successive minima or maxima in the pattern, the phase velocity and hence the effective index is easily determined. It can be seen that the effective (phase) index is $<1$, similar to the case of a parallel conducting plate waveguide of which the dispersion curve is shown for comparison. The resulting curves in the right-hand side of fig. 2 show a systematic $5 . .12 \%$ difference between the results obtained using different calculation methods. This is due to the so-called numerical dispersion of the algorithms, which scales with the square of the calculation grid interval (in these calculations the grid was $12 \times 12$ per unit cell of the crystal). 

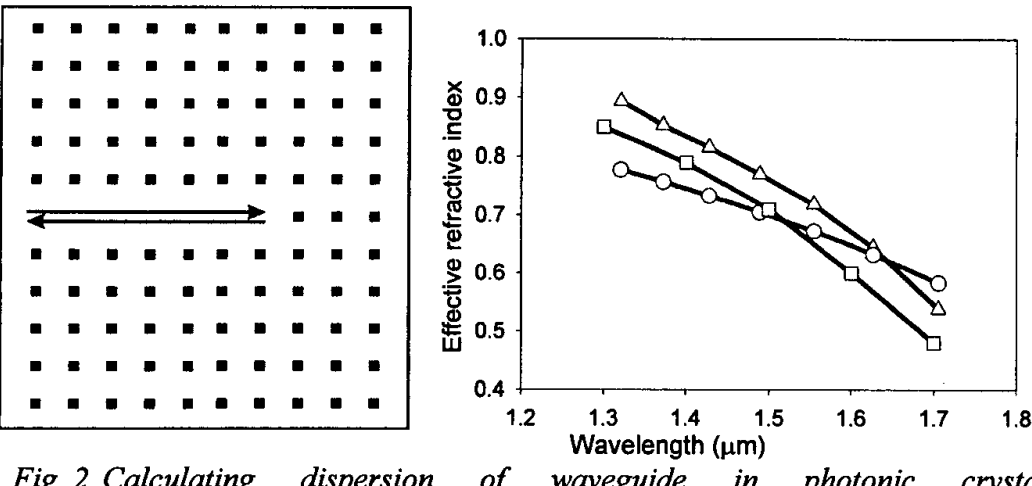

Fig. 2 Calculating dispersion of waveguide in photonic crystal. Left: waveguide in crystal with arrows indicating incident and reflected waves. Right: calculated effective index vs. wavelength (triangles FDTD; squares Helmholtz solver; circles parallel conducting plates waveguide).

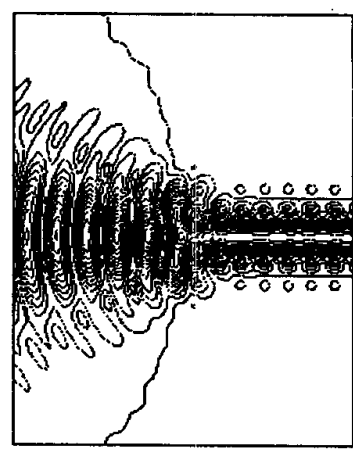

Fig. 3 Coupling of $a$ Gaussian beam to a channel in a photonic crystal.

An important problem of practical devices is the coupling between photonic-crystal based waveguides and external free-space beams or conventional waveguides. Figure 3 shows the coupling of a free-space gaussian beam $(\lambda=1.5$ $\mu \mathrm{m})$, focused to a $1.0 \mu \mathrm{m}$ waist at the entrance of a channel defined by removing one row of rods. The contour plot, obtained using the Helmholtz solver, shows lines of equal wave amplitude. It can be seen that due to partial reflection a standing wave pattern arises in the free space region (left part of plot). It is also interesting to see that the guided wave in the channel (right) penetrates only into the first few layers of the photonic crystal. This evanescent field is concentrated around the high-index rods. The calculated coupling loss is $50.5 \%$. Figure 3 suggests that a reduced beam waist will lead to a better coupling efficiency.

In fig. 4, we show coupling from a conventional dielectric slab waveguide (slab width $0.5 \mu \mathrm{m}$, refractive index 1.45, fundamental mode at $\lambda=1.5 \mu \mathrm{m})$. Again, we observe a partially standing wave in the dielectric slab, caused by reflection at the junction. In this, non-optimized, configuration, the calculated coupling loss is $24.7 \%$.

In summary: We developed numerical tools based on the finite-difference timeddomain (FDTD) method and a pandirectional planar Helmholtz solver, which we applied to modeling structures in a 2-dimensional photonic crystal. We calculated the bandgap of the crystal and the dispersion of a waveguide that is formed by a missing row of rods in the crystal. The coupling to this waveguide of both a Gaussian

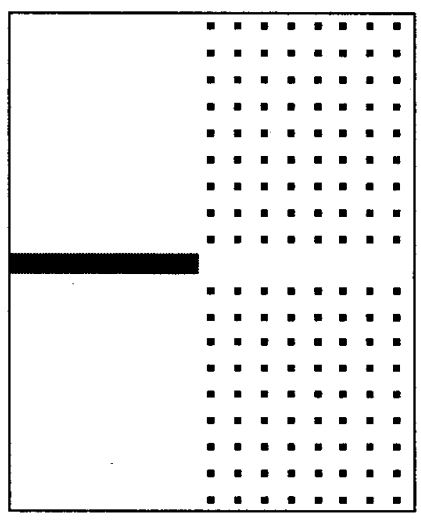

Fig. 4 Coupling a dielectric slab waveguide to a channel in a photonic crystal. Left: configuration; right: calculated field amplitude. beam and a dielectric slab waveguide could be modeled and coupling efficiencies can be calculated.

Acknowledgement: Part of this work was funded by the Dutch Technology Foundation STW.

\section{References}

[1] J.D. Joannopoulos, R.D. Meade, J.N. Winn, "Photonic crystals: molding the flow of light", Princeton Univ. Press, 1995.

[2] A. Mekis, J.C. Chen, I. Kurland, S.H. Fan, P.R. Villeneuve, J.D. Joannopoulos, Phys. Rev. Lett., 77, 3787, 1996.

[3] H.J.W.M. Hoekstra, OQE, 29, 157, 1997.

[4] A. Taflove, "Computational Electrodynamics: The Finite-Difference Time-Domain Method", Artech House, Boston London, 1995.

[5] R. Stoffer, H.J.W.M. Hoekstra, E. van Groesen, F.P.H. van Beckum, Proc. ECIO'99, Torino / Italy, 13/4/99, 47, 1999.

[6] J.P. Bérenger, J. Comput. Phys., 134, 185, 1994. 